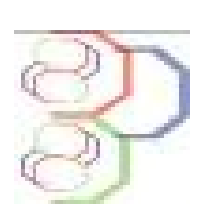

\title{
Digestibilité in vivo de trois types de rations alimentaires en vrac à base de fourrages verts, produits et sous-produits de maïs chez l'aulacode d'élevage au Benin
}

\author{
AïZOUN Finagnon Frédéric ${ }^{1,2^{*}}$, POMALEGNI Sètchémè Charles Bertrand ${ }^{1}$, GBEMAVO Dossou Seblodo \\ Judes Charlemagne ${ }^{3}$, FAROUGOU Souaïbou ${ }^{2}$, YOUSSAO Issaka ${ }^{4}$, MENSAH Guy Apollinaire ${ }^{1}$ \\ ${ }^{1}$ Laboratoire des Recherches Zootechniques, Vétérinaire et Halieutique (LRZVH), Institut National des Recherches \\ Agricole du Bénin (INRAB), 01 BP 884 Recette Principale, Cotonou 01 République du Bénin. \\ 2Département de Production et Santé Animales, Unité de Recherches en Biotechnologie de la Production et de la Santé \\ Animales (URBPSA), École Polytechnique d'Abomey-Calavi (EPAC), 01 BP 2009 Cotonou, Bénin. Université d'Abomey- \\ Calavi. \\ ${ }^{3}$ Laboratoire de Biomathématiques et d'Estimations Forestières (LABEF), Faculté des Sciences Agronomiques, Université \\ d'Abomey-Calavi, 04 BP 1525, Cotonou, Bénin. \\ ${ }^{4}$ Université d'Abomey-Calavi (UAC), École Polytechnique d'Abomey-Calavi (EPAC), Département de Production et Santé \\ Animales, Laboratoire de Recherches en Biologie Appliquée (LARBA), 01 BP 2009 Cotonou, Bénin. \\ * Auteur correspondant, E-mail : fridness2009@yahoo.fr, Tél : (+229) 97652026 / 64114608
}

Original submitted in on $1^{\text {st }}$ February 2017. Published online at www.m.elewa.org on 30 th April 2017 https://dx.doi.org/10.4314/jab.v112i1.14

\section{RÉSUMÉ}

Objectif : Au Bénin, l'aulacodiculture constitue un acquis important en matière d'élevage des espèces animales non conventionnelles. L'objectif est d'évaluer la digestibilité in vivo de 3 rations alimentaires à base de fourrages verts, produits et sous-produits de maïs et autres ingrédients alimentaires chez l'aulacode d'élevage. Méthodologie et Résultats : 24 aulacodes d'élevage âgés de cinq mois repartis en trois lots de huit ont été utilisés et installés dans des cages de digestibilité. La consommation alimentaire moyenne a varié de 59,19 \pm $8,80 \mathrm{~g}$ à $145,67 \pm 6,44 \mathrm{~g}$ pour les trois rations. Le CUDa en \% de la MS a varié entre 78,10 $\pm 0,01$ et 82,19 \pm 0,02 . Celui de la protéine a varié de $87,57 \pm 5,69$ à $90,83 \pm 0,03 \%$ et celui de fibres brutes estimées a varié de $74,51 \pm 0,07$ à $78,44 \pm 0,03 \%$ pour les trois rations.

Conclusions et Application : La ration 2 constituée de fourrages verts, de produits et sous-produits de maïs et autres ingrédients alimentaires est la plus consommée et la plus digérée par les aulacodes d'élevages et la ration 3 constituée des produits et sous-produits de maïs est la moins digérée. La ration alimentaire 2 peut être utilisée par les éleveurs d'aulacode pour nourrir les animaux afin d'améliorer la digestibilité et les performances pour une production durable de l'aulacodiculture.

Mots clés : Aulacodiculture, Alimentation, Coefficient d'utilisation digestif apparent, Maïs, Bénin. 
Aizoun et al., J. Appl. Biosci. 2017 Digestibilité in vivo de trois types de rations alimentaires en vrac à base de fourrages verts, produits et sous-produits de maïs chez l'aulacode d'élevage au Benin.

In vivo digestibility of three type rations based from green fodders, products and by-products of maize to the grasscutter in Benin

\begin{abstract}
Objectives: In Benin, grass-cutter breeding constitutes an important achievement in breeding unconventional animal species. The objective is to evaluate the in vivo digestibility of 3 food rations at based on green fodders, products and by-products of maize and other food ingredients in the grasscutters in captivity.

Methodology and Results: 24 grasscutters breeding aged five months divided into three lots of eight were installed in digestibility cages. The average food consumption ranged from $59.19 \pm 8.80 \mathrm{~g}$ to $145.67 \pm 6.44 \mathrm{~g}$ for the three rations. The apparent digestive utilisation coefficient (CUDa) in percentage for dry matter was from $78.10 \pm 0.01$ and $82.19 \pm 0.02$. That of the protein varied from $87.57 \pm 5.69$ to $90.83 \pm 0.03 \%$ and the estimated crude fibre ranged from $74.51 \pm 0.07$ to $78.44 \pm 0.03 \%$ for three rations.

Conclusions and Application: The ration 2 consists of green fodders, products and by-products maize and other food ingredients is the most consumed and digested by of the grasscutters and ration 3 consists of products and by-products of maize is the least. The food ration 2 can be used by the grasscutters breeders for animal feed to improve the digestibility and performance for sustainable production of grasscutters.

Key words: Feeding, apparent digestive utilisation coefficient, maize, Benin.
\end{abstract}

\section{INTRODUCTION}

L'aulacode (Thryonomys swinderianus, Temminck, 1827) est un rongeur hystricomorphe dont l'élevage se développe davantage en Afrique de l'Ouest et du Centre pour des raisons à la fois de spéculation alimentaire et de gestion des ressources fauniques (Jori et al., 1995). Sa demande de plus en plus forte, est estimée à 80 millions de têtes d'aulacodes soit 300.000 tonnes de viande consommées par an dans l'ensemble dans les pays d'Afrique de l'Ouest (Mensah, 1993; Fantodji et Mensah, 2000). Son élevage en plein essor dans de nombreux pays africains, fait l'objet d'études et d'expérimentations (Mensah, 2000). Ainsi plusieurs auteurs ont montré la possibilité de nourrir les aulacodes d'élevage avec des granulés de fourrages verts rapportés par Aïzoun et al. (2015) et Pomalegni et al. (2015). Quel que soit le fourrage graminéen ou aérien, l'aulacode consomme les tiges, l'écorce des troncs et finalement quelques feuilles. Ce comportement alimentaire qui consiste à trier entre les particules de l'aliment est une aide précieuse pour l'auto-usure de ses incisives, mais fait de cet animal un gaspilleur de $70 \%$ des fourrages qui lui sont servi (Mensah et al., 1996). La productivité de cet animal est limitée par la cherté des ingrédients alimentaires, la qualité nutritionnelle et la disponibilité des aliments utilisés.
En saison pluvieuse, les fourrages avec un taux élevé en protéine et une bonne digestibilité sont disponibles, mais deviennent pauvres en qualité en saison sèche (Ansah et al., 2012). Au Bénin, la production de maïs ne cesse de croître avec 931.599 tonnes en 2007 et une estimation de 1.900.000 tonnes en 2015 (PSRSA, 2010). Ceci induit un accroissement et une disponibilité des produits et sous-produits de cette céréale qui ne sont pas suffisamment valorisés dans l'alimentation des aulacodes. La digestibilité indique le degré d'utilisation des aliments ou nutriments par l'animal. L'addition de maïs ou l'utilisation de granulés augmente la consommation alimentaire et améliore la digestibilité Sagbo (1985). Or une bonne digestibilité apparente alimentaire de concentrés, bien que les fourrages soient indispensables dans l'alimentation de l'aulacode, conduit à des meilleures performances zootechniques (Mensah et al., 1992). D'où l'intérêt d'étudier la digestibilité des rations alimentaires formulées à base des fourrages verts et des produits et sous-produits du maiis et autres ingrédients alimentaires chez les aulacodes d'élevage. L'hypothèse de recherche testée est : La consommation alimentaire et la digestibilité sont fonction des rations alimentaires. 

de fourrages verts, produits et sous-produits de maïs chez l'aulacode d'élevage au Benin.

\section{MATÉRIELS ET MÉTHODES}

Milieu d'étude : La présente étude s'est déroulée dans l'aulacoderie du Sous-Programme Élevage des Espèces Animales Non Conventionnelles (S-PEEANC) du Laboratoire de Recherches Zootechnique, Vétérinaire et Halieutique (LRZVH), du Centre de Recherches Agricoles de l'Institut National de Recherches Agricoles du Bénin (INRAB) situé dans la Commune d'Abomey-Calavi. Le site abrite un climat de type guinéen, avec deux saisons sèches (mi-novembre à mi-mars et mi-juillet à miseptembre) et deux saisons pluvieuses (mi-mars à mijuillet et mi-septembre à mi-novembre). La pluviométrie moyenne est de $1.200 \mathrm{~mm}$ par an et les températures moyennes mensuelles varient entre 27 et $31^{\circ} \mathrm{C}$ avec une humidité relative de l'air fluctuant entre $65 \%$ de janvier à mars et $97 \%$ de juin à juillet. La moyenne mensuelle variait entre 27 et $31^{\circ} \mathrm{C}$ avec un écart de $3,2^{\circ} \mathrm{C}$ entre Mars et Août.

Matériel végétal et animal : Trois rations alimentaires ont été formulées et utilisées dans le cadre de cette étude. La ration $\mathrm{R} 1$ était constituée de fourrages verts, la deuxième ration alimentaire (R2) comportait des fourrages verts et compléments alimentaires et la troisième ration alimentaire $(R 3)$ était constituée de produits et sous-produits de maïs. Les ingrédients alimentaires qui ont servi à la formulation des rations alimentaires ainsi que les compositions centésimales des trois rations sont présentés dans le tableau 1. L'essai a été réalisé sur 24 aulacodes d'élevage âgés de 5 mois. Ces animaux ont été répartis en 3 lots de 8 aulacodes en fonction du poids des animaux avec des poids vifs corporel de $944,11 \pm 70,66 \mathrm{~g} ; 1066,67 \pm 199,85 \mathrm{~g}$ et $1054,44 \pm 41,98 \mathrm{~g}$ respectivement. Des cages de digestibilité en bois de dimensions $0,6 \mathrm{~m} \times 0,5 \mathrm{~m} \times 0,4 \mathrm{~m}$ recouvert de grillage ont été utilisées. Chaque cage était munie d'une petite porte et d'un dispositif de collecte de fèces composé d'un tiroir. Elle comportait une mangeoire et un abreuvoir en ciment. Les espèces fourragères utilisées (tableau 1) dans la formulation des rations expérimentales ont été fauchées la veille et séchées au soleil toutes la journée du lendemain afin de réduire leur charge parasitaire.

Tableau 1 : Composition centésimale des rations alimentaires expérimentales

\begin{tabular}{lccc}
\hline Ingrédients alimentaires & \multicolumn{3}{c}{ Proportion des rations (\%) } \\
\cline { 2 - 4 } & R1 & R2 & R3 \\
\hline Panicum maximum & 30,00 & 25,00 & - \\
Pennisetum purpureum & 25,00 & 25,00 & - \\
Paspalum vaginatum & 20,00 & 20,00 & - \\
Leucaena leucocephala & 4,00 & 4,00 & 4,00 \\
Moringa oleifera & 4,00 & - & 4,00 \\
Imperata cylindrica & 5,00 & - & - \\
Grains de maïs & - & 10,00 & 28,50 \\
Son de maïs & - & - & 30,00 \\
Farine de maïs & - & - & 14,00 \\
Son de riz & - & 3,50 & 10,00 \\
Son de blé & - & 4,00 & - \\
Tourteaux de coton & - & 3,00 & - \\
Tourteaux de soja & - & 3,50 & - \\
spathe de maïs & - & - & 7,50 \\
Cossette de manioc & 10,00 & - & - \\
Poudre de coquille d'huître & 1,50 & 1,50 & 1,50 \\
Sel de cuisine & 0,50 & 0,50 & 0,50 \\
\hline Total & 100 & 100 & 100 \\
\hline
\end{tabular}

Expérimentations : Chaque lot d'aulacode a été soumis à trois séries d'essai qui correspondent aux trois traitements. La distribution des aliments s'est effectuée deux fois par jour le matin entre $7 \mathrm{~h} 30$ et $8 \mathrm{~h}$ et le soir entre $16 \mathrm{~h} 30$ et $17 \mathrm{~h}$. L'eau était servie dans les abreuvoirs une fois dans la journée à volonté. Une phase d'adaptation à la cage de digestibilité et à l'alimentation a été observée pendant 10 jours, suivie d'une période de collecte de données de 10 jours. Une balance de précision de marque Kitchen d'une portée de $5 \mathrm{~kg}$ et de précision $1 \mathrm{~g}$ a été également utilisée pour la pesée des différents échantillons. 

de fourrages verts, produits et sous-produits de maïs chez l'aulacode d'élevage au Benin.

Les données collectées : Durant l'expérimentation les quantités d'aliments distribués, du refus alimentaire et de matière fécale excrétée ont été pesées chaque jour. Les formules mathématiques utilisées pour calculer les variables étudiées à la fin de l'expérimentation sont présentées (Tableau 2).

Tableau 2 : Formules mathématiques utilisées pour calculer les variables étudiées

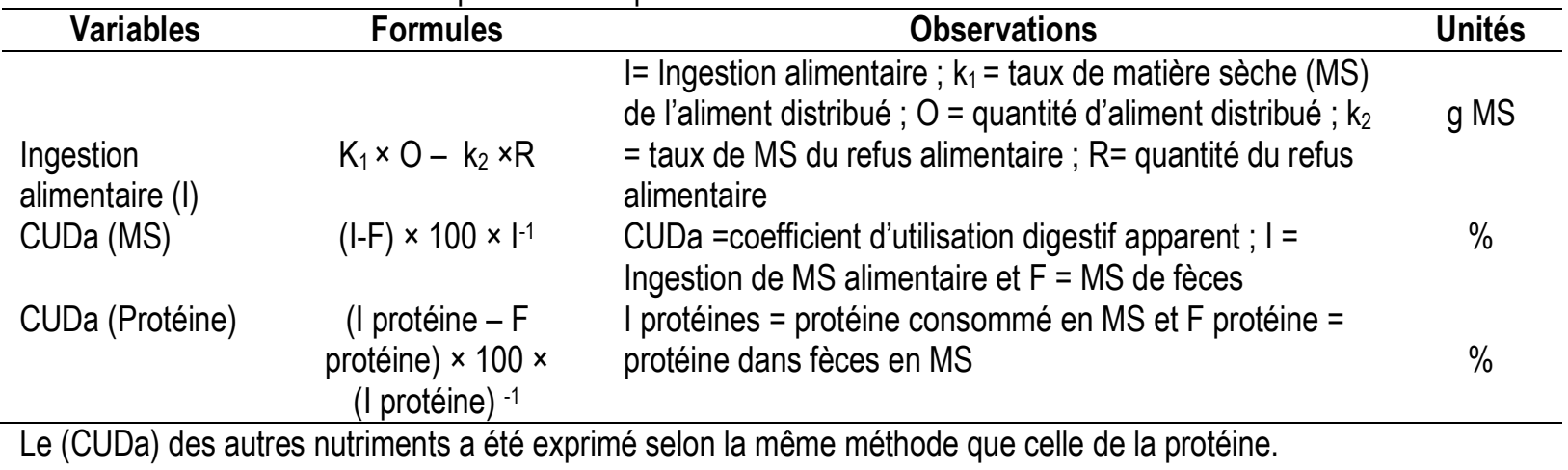

Analyses de laboratoire : Des échantillons d'aliments distribués, d'aliments refusés et de matière fécale ont été prélevés et analysés. Les analyses ont été faites suivant les méthodes admises par le Bureau Interprofessionnel d'Études Analytiques (BIPEA, 1976) et de l'Association of Official Analytical Chemist (AOAC, 2000). Les analyses ont été effectuées au Laboratoire des Sciences du Sol, Eaux et Environnement (LSSEE) du Centre de Recherches Agricoles (CRA) d'Agonkamey de l'Institut National des Recherches Agricoles du Bénin (INRAB) et au Laboratoire des Sciences et Techniques de Production Animale de la Faculté des Sciences Agronomiques (FSA) de l'Université d'Abomey-Calavi (UAC). La détermination de la matière sèche (MS) a été faite par séchage en mettant à l'étuve à $105^{\circ} \mathrm{C}$ pendant $24 \mathrm{~h}$ jusqu'à la stabilisation du poids puis pesé après l'avoir laissé refroidir. Ceci pour extraire tout résidu d'eau. La détermination de la teneur en protéine brute a été faite par la méthode de Kjeldahl (AOAC, 2000), celle des fibres brutes par la méthode de Van Soest et al. (1991), celle des matières grasses a été déterminée par le dispositif de

\section{RÉSULTATS}

Valeurs nutritives des rations alimentaires: La composition chimique des rations est présentée dans le tableau 3. L'analyse des résultats a montré que les taux de matières sèches, des protéines brutes, de la matière grasse, des fibres brutes, des Cendres brutes sont
Soxhlet (AOAC, 2000) et celle des cendres brutes par carbonisation lente au four. Les teneurs en calcium et en phosphore sont dosées par spectrophotométrie d'absorption atomique.

Analyses statistiques des données: Les données collectées ont été saisies et enregistrées sur un tableau Excel 2010. La statistique descriptive a été réalisée en termes de moyenne et d'écart type pour les données. Le test de normalité de Royan-Joiner et le test d'égalité des variances ont été exécuté pour tester respectivement la normalité et l'égalité des variances. En cas de normalité, une analyse de variance (ANOVA) a été effectuée pour les CUDa et la consommation alimentaire. Dans le cas contraire, c'est le test non paramétrique de Kuskal-Wallis qui a été faite. En cas de différences significatives entre les moyennes pour un paramètre suivant les trois rations le test de séparation des moyennes de Student Newman \& Keuls a été réalisé au seuil de $5 \%$. Les analyses ont été réalisées avec le logiciel R3.0.2 (R Development Core Team. 2012., http://www.Rproject.org/)

significativement différents entre les trois rations alimentaires $(p<0,001)$. Par contre les taux de matière organique, Calcium et Phosphore ne varient pas significativement entre les trois rations alimentaires $(p>0,05)$. 

de fourrages verts, produits et sous-produits de maïs chez l'aulacode d'élevage au Benin.

Tableau 3 : Composition chimique des rations alimentaires.

\begin{tabular}{lllll}
\hline & \multicolumn{2}{c}{ Composition chimique des rations alimentaires } & \\
\cline { 2 - 4 } \multicolumn{1}{c}{ Paramètres } & \multicolumn{1}{c}{ Ration 1 } & Ration 2 & Ration 3 & Prob \\
\hline Matière Sèche $(\%)$ & $87,46 \pm 0,01 \mathrm{~b}$ & $89,25 \pm 0,01 \mathrm{a}$ & $85,91 \pm 0,01 \mathrm{c}$ & $1,38 \mathrm{e}-07$ \\
Matière Organique $(\% \mathrm{MS})$ & $91,11 \pm 0,01 \mathrm{a}$ & $90,95 \pm 0,01 \mathrm{a}$ & $90,26 \pm 0,01 \mathrm{a}$ & 0,1017 \\
Protéine brute $(\% \mathrm{MS})$ & $14,26 \pm 0,01 \mathrm{~b}$ & $18,50 \pm 0,00 \mathrm{a}$ & $12,5 \pm 0,01 \mathrm{c}$ & $1,69 \mathrm{e}-08$ \\
Matière grasse $(\% \mathrm{MS})$ & $2,75 \pm 0,00 \mathrm{~b}$ & $2,24 \pm 0,01 \mathrm{c}$ & $3,85 \pm 0,01 \mathrm{a}$ & $7,97 \mathrm{e}-05$ \\
Fibre brute $(\% \mathrm{MS})$ & $27,45 \pm 0,07 \mathrm{~b}$ & $25.30 \pm 0,00 \mathrm{c}$ & $31,85 \pm 0,01 \mathrm{a}$ & $1,21 \mathrm{e}-06$ \\
Cendre bute $(\% \mathrm{MS})$ & $8,91 \pm 0,07 \mathrm{c}$ & $9,40 \pm 0,00 \mathrm{~b}$ & $9,57 \pm 0,01 \mathrm{a}$ & $2,98 \mathrm{e}-06$ \\
Calcium $(\% \mathrm{MS})$ & $0,65 \pm 0,02 \mathrm{a}$ & $0,66 \pm 0,02 \mathrm{a}$ & $0,64 \pm 0,05 \mathrm{a}$ & 0,2679 \\
Phosphore $(\% \mathrm{MS})$ & $0,33 \pm 0,04 \mathrm{a}$ & $0,36 \pm 0,05 \mathrm{a}$ & $0,40 \pm 0,02 \mathrm{a}$ & 0,0969
\end{tabular}

Les valeurs suivies des lettres différentes $(a, b, c)$ sur la même ligne sont significativement différentes suivant le test de Student Newman \& Keuls au seuil de $5 \%$. Prob = Probabilité.

La consommation alimentaire et la digestibilité des rations : La consommation alimentaire journalière et les coefficients d'utilisation digestifs apparents ont été consignés dans le tableau 4. La plus forte consommation alimentaire quotidienne a été obtenue chez les animaux nourris avec la ration $\mathrm{R} 2(145,67 \pm 6,44 \mathrm{~g})$ et la plus faible $(59,19 \pm 8,80 \mathrm{~g})$ a été enregistrée chez les animaux qui avaient consommés la ration R3. La différence entre la consommation alimentaire moyenne quotidienne des rations a été significative $(p<0,001)$. Le CUDa de tous les nutriments des rations alimentaires s'est élevé à plus de
$74 \%$. Le CUDa des matières sèches, des matières organiques, des protéines brutes et des matières grasses étaient supérieurs à $80 \%$ sauf celui de la MS de la ration 3 . La différence n'a pas été significative $(p>0,05)$ entre les CUDa des nutriments des protéines brutes, fibres brutes et cendres des trois rations. Par contre la différence a été significative $(p<0,05)$ entre les CUDa de la matière sèche, matière organique et matière grasse des trois rations. Les valeurs moyennes de CUDa les plus élevées étaient enregistrées pour les protéines brutes $(90,83 \pm 0,03)$ et les matières grasses $(92,17 \pm 0,29)$ de la ration $R 2$.

Tableau 4 : Consommation alimentaire journalière et Coefficient d'Utilisation Digestif apparent (CUDa) des nutriments des rations expérimentales.

\begin{tabular}{lllll}
\hline Ingestion alimentaire et CUDa & \multicolumn{1}{c}{ R1 } & \multicolumn{1}{c}{ R2 } & \multicolumn{1}{c}{ R3 } & \multicolumn{1}{c}{ Prob } \\
\hline Ingestion alimentaire & $144,15 \pm 4,12 \mathrm{a}$ & $145,67 \pm 6,44 \mathrm{a}$ & $59,19 \pm 8,80 \mathrm{~b}$ & 0,001614 \\
Matière Sèche & $80,12 \pm 0,01 \mathrm{~b}$ & $82,19 \pm 0,02 \mathrm{a}$ & $78,10 \pm 0,01 \mathrm{c}$ & $2,71 \mathrm{e}-07$ \\
Matière Organique & $82,77 \pm 0,01 \mathrm{~b}$ & $84,28 \pm 0,01 \mathrm{a}$ & $80,82 \pm 0,01 \mathrm{c}$ & $4,4 \mathrm{e}-08$ \\
Protéine brute & $88,74 \pm 0,06$ & $90,83 \pm 0,03$ & $87,57 \pm 5,69$ & 0,647 \\
Matière grasse & $89,09 \pm 00 \mathrm{~b}$ & $92,17 \pm 0,29 \mathrm{a}$ & $85,84 \pm 0,44 \mathrm{c}$ & 0,000579 \\
Fibre brute & $78,05 \pm 0,08$ & $78,44 \pm 0,03$ & $74,51 \pm 0,07$ & 0,1017 \\
Cendre bute & $80,34 \pm 0,81$ & $80,42 \pm 0,01$ & $79,11 \pm 0,01$ & 0,113 \\
\hline
\end{tabular}

Les valeurs suivies des lettres différentes $(a, b, c)$ sur la même ligne sont significativement différentes suivant le test de Student Newman \& Keuls au seuil de 5\%. Prob = Probabilité.

\section{DISCUSSION}

Composition chimique : L'analyse des résultats montre que le taux de matière sèche, de protéine brute, de matière grasse, de fibre brute, Cendre brute est significativement différent entre les trois rations alimentaires $(p<0,001)$. Par contre le taux de la matière organique, de calcium et de phosphore n'est pas significativement différent entre les trois rations alimentaires $(p>0,05)$. Les teneurs en matières sèches obtenues dans cette étude sont similaires à celles trouvées dans une étude de l'influence de la forme physique des aliments sur la croissance et le rendement en carcasse de Thryonomys swinderianus (Traoré et al., 2009) ; à celles trouvées par Poku et al. (2013) dans une expérimentation sur la croissance, la reproduction et les caractéristiques des carcasses des aulacodes nourris avec un aliment à différent taux de protéine. En revanche, elles sont inférieures à celles obtenues par Etchu et al. (2012) dans une étude comparative de performance de l'aulacode nourris avec du maïs et des granulés de rongeurs au Caméroun. Les teneurs de protéine brute des 3 rations variant de 12,5 à $18,5 \%$ obtenues au cours de cette expérimentation appartiennent à l'intervalle 12- 
$18,5 \%$ et $14-18 \%$ indiquées pour les aulacodes (Mensah, 1993 ; Mensah, 1995). Les teneurs de matière grasse obtenues pour les trois rations alimentaires expérimentales sont comprises entre 2,5-4,5\% indiquées (Mensah, 1993 ; Mensah, 1995).

Consommation alimentaire quotidienne des aulacodes : La consommation alimentaire a pour but de satisfaire les besoins alimentaires et obtenir une meilleure croissance et production chez l'animal. Les 3 consommations alimentaires journalières moyennes obtenues dans cette étude appartiennent à l'intervalle 40,1 à 153,9 g MS/ kg PV rapportée par Traoré et al. (2009), chez des aulacodes nourris avec une ration en vrac. La consommation alimentaire quotidienne des trois rations alimentaires obtenues au cours de cet essai est inférieure à l'intervalle 150-250 g obtenue par Yeboah et Adamu (1995). Dans une étude de performance de croissance de l'aulacode nourris avec un aliment avec différents sources de fibre, Uwalaka et Ahaotu (2013) ont obtenu une consommation variant entre 102,74-116,31 g MS. Ansah et al. (2012) au cours d'une expérimentation sur l'évaluation de l'épi de maïs au Ghana sur la performance de croissance de l'aulacode ont rapporté une consommation quotidienne de 114 et $115 \mathrm{~g}$. Ogunjobi et al. (2014) ont trouvé une consommation alimentaire quotidienne qui varie entre $105,14 \mathrm{~g}$ et $107,23 \mathrm{~g}$ chez des aulacodes dans une étude sur la performance de croissance des aulacodes mâles juvénile d'élevage nourris avec deux fourrages courants. Ces résultats sont inférieurs à ceux obtenus dans le présent travail pour la consommation alimentaire quotidienne de la ration 1 et la ration 2. Par contre ces résultats sont supérieurs à celui trouvé avec la ration 3 .

Digestibilité alimentaire des nutriments : Lors d'une évaluation de la qualité protéinique de différentes variétés de maïs au Nigéria, les valeurs moyennes de CUDa de la MS comprises entre $78,23 \%$ et $79,66 \%$ sont rapportées (Ajasin et al., 2014). De même, Ogunjobi et al. (2014) ont trouvé les valeurs moyennes de CUDa de la MS variant entre $80,75 \pm 0,72 \%$ et $83,95 \pm 0,72 \%$. Traoré et al. (2008) et Traoré et al. (2009 ont trouvé respectivement pour les CUDa de la MS des valeurs comprises entre $(76,3 \pm 42,8 \%$ et $85,3 \pm 4,7 \%)$ et entre $(79,25 \pm 5,80 \%$ et $93,13 \pm 3,76 \%)$. Ces valeurs appartiennent à ses intervalles. Par contre les valeurs que nous avons déterminées sont supérieures aux CUDa de la MS variant entre 66-68\% trouvés par Obi et al. (2008) et entre 73,40 $\%$ et $74,04 \%$ rapportés par Uwalaka et Ahaotu (2013). Mais Ces valeurs sont inférieures à celles rapportées par Zyl van et Delport (2010) comprises entre 82,8 $\pm 8,1 \%$ et $91,7 \%$ de CUDa de la MS.
Les valeurs moyennes des CUDa de la protéine brute obtenues dans cette étude sont similaires à : celles trouvées par Traoré et al. (2008) comprises entre 81,4 \pm $2,1 \%$ et $91,8 \pm 1,9 \%$; à celles rapportées par Karikari et Nyameasem (2009) variant entre $84,8 \pm 0,78 \%$ et $95,5 \pm$ $1,00 \%$. Ces valeurs concordent également avec celles obtenues par Traoré et al. (2009) comprises entre 88,30 $\pm 30 \%$ et $89,23 \pm 4,32 \%$; celles trouvées par Uwalaka et Ahaotu (2013) variant entre $82,21 \%$ et $86,20 \%$ et aux valeurs obtenues par Wogar et Ayara (2015) comprises entre $85,50 \%$ et $90,80 \%$. Par contre elles sont supérieures à celles obtenues par Ogunjobi et al. (2014) variant entre $80,67 \pm 0,78 \%$ et $83,47 \pm 0,78 \%$. Les valeurs que nous avons déterminées sont également supérieures aux valeurs de CUDa de protéine trouvée par Obi et al. (2008) variant entre $57-59 \%$; à celles rapportées par Ansah et al. (2012) comprises entre 67,7 $\pm 2,37 \%$ et $69,5 \pm 6,52 \%$.

Les valeurs moyennes des CUDa de la matière grasse obtenues dans cette étude sont en accord avec celles rapportés par Traoré et al. (2008) comprises entre 86,5 \pm $4,3 \%$ et $93,3 \pm 1,6 \%$ de même que celles trouvées par Traoré et al. (2009) variant entre $88,13 \pm 4,57 \%$ et 91,56 $\pm 4,28 \%$. Les valeurs des CUDa de fibre brute rapportées par Traoré et al. (2008), Traoré et al. (2009) et par Uwalaka et Ahaotu (2013) variant respectivement entre $75 \pm 2,9 \%$ et $85,9 \pm 4,5 \% ; 76,21 \pm 4,26 \%$ et $76,59 \pm 4,95 \%$ puis $76,96 \%$ et $78,12 \%$ obtenues par Uwalaka et Ahaotu (2013) sont similaires aux valeurs moyennes obtenues dans cette étude. Par contre, ces valeurs moyennes de fibre brute obtenues dans cette étude sont inférieures à celles obtenues par Ogunjobi et al. (2014); par Wogar et Ayara (2015) qui varient respectivement entre $80,39 \pm 0,37 \%$ et $82,74 \pm 0,40 \%$ puis $82,80 \%$ et $85,60 \%$. Par ailleurs, elles sont supérieures à celles obtenues par Ajasin et al. (2014) et qui varient entre $69,78 \%$ et $73,87 \%$. Les valeurs des CUDa de cendre brute obtenues dans cette étude concordent avec celles obtenues par Uwalaka et Ahaotu (2013) comprises entre $73,60 \%$ et $80,77 \%$ de même que celles rapportées par Ogunjobi et al. (2014) qui ont varié entre $80,31 \pm 0,19 \%$ et $80,91 \pm 0,20 \%$. Par contre ces valeurs sont inférieures à celles rapportées par Wogar et Ayara (2015) qui varient entre $83,50 \%$ et $86,20 \%$; à celles rapportées par Traoré et al. (2009) variant entre $80,43 \pm 5,46 \%$ et $83,60 \pm 4,68 \%$; mais supérieures à celles trouvées par Ansah et al. (2012) qui varient entre $13,06 \pm 9,9 \%$ et $51,7 \pm 2,89 \%$. Les valeurs moyennes des CUDa de la matière organique obtenues dans cette étude sont en accord avec celles trouvées par Traoré et al. (2008) variant entre $76,2 \pm 2,7 \%$ et $85,2 \pm 4,7 \%$ de 

de fourrages verts, produits et sous-produits de maïs chez l'aulacode d'élevage au Benin.

même que celles rapportées par Traoré et al. (2009) variant entre $82,97 \pm 6,45 \%$ et $85,95 \pm 1,25 \%$. Au regard des résultats obtenus dans cette étude, la ration 2 est la plus digérée et la ration $\mathrm{R} 3$ la moins digérée. Ce qui est en accord avec les observations faites par Van Zyl et

\section{CONCLUSION}

Au terme de l'étude, l'ensemble des résultats obtenus montrent que les aulacodes d'élevages peuvent être nourris avec des rations alimentaires formulées avec une bonne digestibilité apparente. La consommation et la digestibilité alimentaire chez les aulacodes d'élevage sont fonction des rations alimentaires. . La ration alimentaire R2 composée de fourrages verts, de produits et sousproduits de maïs et autres ingrédients alimentaires est la

\section{RÉFÉRENCES BIBLIOGRAPHIQUES}

Aïzoun FF, Pomalegni SCB, Farougou S. \& Mensah GA, 2015. Synthèse bibliographique sur l'alimentation de l'aulacode avec des granulés de fourrages verts au Bénin. ASA, 19(2) volume special : 389-400. 2015 ISSN 1669 - 5009. 12p.

Ajasin FO, Omole AJ, Okpeze CN, Ononogbo C, Owosibo AO, Odunsi OO, Oladele-Bukola MO, 2014. Evaluation of different varieties of quality protein maize as an energy source in grasscutter diet. Agricultural Science Research Journal 4(7) : 111-114.

Ansah T, Aglolosu AA, Teye GA, Akwasi A, OpokuAgyean M, 2012. Evaluation of Corn Cob on the Growth Performance of Grasscutter (Thryonomys swinderianus). Animal Science and Biotechnologies, 45(1) : 7-10.

AOAC, 2000. Association of Official Analytical Chemist, Official Methods of Analysis 17th edition Washington D.C.

BIPEA, 1976. Bureau Interprofessionnel d'Études analytiques. Recueil des méthodes d'analyses des communautés Européennes : 2 route du port Charbonnier, 92230 Gennevilliers, 140p.

Etchu KA, Ndzi VN, Ndamukong KJ, Oben B, 2012. Comparative performance of grasscutters (Thryonomys swinderianus) fed maize and rodent pellets as concentrate supplement under intensive management system in Cameroon. African Journal of Agricultural Research, 7(6): 883-891.

Fantodji A. et Mensah GA, 2000: Rôle et impact économique de l'élevage intensif de gibier au Bénin et en Côte-d'Ivoire. Actes Séminaire International sur l'élevage intensif de gibier à but al. (1999) qui indique que l'accroissement en taux de fibre dans les rations alimentaires des aulacodes peut être associé à une diminution de la digestibilité de la matière sèche, de protéine et de matière grasse chez les animaux domestiques et à une réduction du taux de croissance.

plus digérée suivie de la ration $\mathrm{R} 1$ constituée de fourrages verts et de la ration R3 composée de produits et sous-produits de maïs. La ration $\mathrm{R} 2$ peut être utilisée dans les aulacodiculture pour améliorer la digestibilité alimentaire chez les animaux conduisant à de meilleures performances zootechniques des aulacodes d'élevages pour le développement durable de l'aulacodiculture.

Alimentaire en Afrique, Libreville 23 et 24 Mai 2000, p25-42.

Jori F, Mensah GA, Adjanohoun E, 1995. Grasscutter farming: an example of rational utilization of wildlife. Biodiversity and Conservation, 4: 257265.

Karikari PK, and Nyameasem JK, 2009. Productive Performance and Carcass Characteristics of Captive Grasscutters (Thryonomys swinderianus) Fed Concentrate Diets Containing Varying Levels of Guinea Grass. World Applied Sciences Journal 6(4): 557-563.

Mensah GA, 1993. Futteraufnahme und verdaulichkeit bein grasnager (Thryonomys swinderianus). Thèse de doctorat. Institut 480, Université de Holenheim, Allemagne, $107 \mathrm{p}$.

Mensah GA, 1995. Consommation et digestibilité alimentaire chez l'aulacode (Thryonomys swinderianus). Tropicultura, 13 (3): 123-124.

Mensah GA, 2000 : Présentation générale de l'élevage d'aulacodes, historique et état de la diffusion en Afrique. Actes Séminaire international sur l'élevage intensif de gibier à but alimentaire à Libreville (Gabon), Projet DGEG/VSF/ADIE/CARPE/UE, pp.45-59.

Mensah GA, Blummel M, Borowy N, Stier CH, Gall CF, 1992. Consommation et digestibilité alimentaire chez l'aulacode (Thryonomys swinderianus). Actes 1ère Conférence Internationale sur l'aulacodiculture PBAA/MDR/BENIN. p. 151-156.

Mensah GA, Schwarzenberg A, Stier CH, Kangni T, Gall $\mathrm{CF}$, 1996. Aspects pratiques en élevage d'aulacodes (Thryonomys swinderianus). Vl. Mesures préventives contre la mauvaise usure 
des incisives. Revue Elévage. et de Médecine Vétérinaire en Pays Tropaux. 49 (4): 341-346.

Obi OO, Omole AJ, Ajasin FO, \& Tewe, 00, 2008. Nutritive potentials of four conventional forages fed to growing grasscutter. Livestock Research for Rural Development, 20 (179).

Ogunjobi JA, Adu BW, Jayeola OB, 2014. Growth performance of captive male grasscutters (Thryonomys swinderianus Temminck 1827) fed two common grasses in Nigeria. International Journal of AgriScience, 4(2): 119-121.

Poku Jnr PA, Annor S Y, and Djang-Fordjour KT, 2013. Growth, Reproduction and Carcass Characteristics of Grasscutters (Thryonomys swinderianus) Fed on Different Levels of Protein Supplement. World Journal of Zoology 8(2): 175184, 2013.

Pomalegni SCB, Babatounde S, Glele Kakaï LR. \& Mensah GA, 2015. Synthèse bibliographique sur l'alimentation, la consommation et la digestibilité alimentaire chez le grand aulacode (Thryonomys swinderianus). Annales des Sciences Agronomiques 19(2): 401-417, 2015. ISSN 1659-5009. 18p.

PSRSA, 2010. Plan Stratégique de Relance du Secteur Agricole au Bénin: Orientations stratégiques et Plan d'action. Version finale, République du Bénin, Cotonou. 110p.

R Core Team, 2012. R: A language and environment for statistical computing. R Foundation for Statistical Computing, Vienna, Austria. URL https://www.Rproject.org/.

Traoré B, Fantodji A, Allou VK, 2008. Digestibilité in vivo chez l'aulacode (Thryonomys swinderianus). Archivos de Zootecnia, 57(218) : 229-234.

Traoré B, Fantodji A, Mensah GA, 2009. Influence de la forme physique des aliments sur la croissance et le rendement en carcasse de Thryonomys swinderianus à trois stades physiologiques. Bulletin de la Recherche Agronomique du Bénin, $\mathrm{N}^{\circ} 65$ septembre 2009, pp. 1-31.

Uwalaka RE and Ahaotu EO, 2013. Performance of Growing grasscutters fed on different fibres sources. International Journal of Veterinary Science 2(3): 85-87. www.ijvets.com.

Van Soest P.J., Robertson J.B. and Lewis B.A. 1991. Methods for dietary fiber, neutral detergent fiber, and non-starch polysaccharides in relation to animal nutrition. Journal of Dairy Science, 74: 3583-3597.
Van Zyl A, Meyer AJ, \& Merwe VM. 1999. The influence of fibre in the diet on growth rates and digestibility of nutrients in the greater cane rat (Thryonomys swinderianus). Comparative Biochemistry and Physiology, Part A: Molecular and Integrative Physiology., 123(2): 129-135.

Wogar GSI and Ayara TE, 2015. Performance of Growing Grasscutters fed a concentrate diet without supplementation. American Journal of Experimental Agriculture, 8(4): 253-260.

Yeboah S and Adamu. 1995. The Cane Rat. Biologist, 42: 86-87.

Zyl van A \& Delport JH, 2010. Digestibility of nutrients and aspects of the digestive physiology of the greater cane rat, Thryonomys swinderianus in two seasons. African Zoology, 45(2): 254-264. 